\title{
Two-Dimensional Frequencies Estimation Using Two-Stage Separated Virtual Steering Vector-Based Algorithm
}

\author{
Ding Liu and Junli Liang \\ School of Automation \& Information Engineering, Xi'an University of Technology, \\ Xi'an 710048, China \\ Correspondence should be addressed to Junli Liang, heery_2004@hotmail.com
}

Received 10 May 2010; Accepted 25 January 2011

Academic Editor: Laurence Mailaender

Copyright ( $\odot 2011$ D. Liu and J. Liang. This is an open access article distributed under the Creative Commons Attribution License, which permits unrestricted use, distribution, and reproduction in any medium, provided the original work is properly cited.

In this paper, we develop a novel two-stage separated virtual steering vector- (SVSV-) based algorithm without association operation to estimate $2 \mathrm{D}$ frequencies. The key points of this algorithm are (i) in the first stage, this paper rearranges the measurement data as virtual rectangular array data matrix and obtains the propagator from the data matrix using least-squares operator. In addition, the virtual steering vector can be separated into two parts using the introduced electric angle that combines $2 \mathrm{D}$ frequencies (to avoid incorrect association especially when multiple 2D frequencies have the same frequency at some dimension), and thus the electric angle and the first part of separated steering vector can be estimated using the derived rank-reduction propagator method; (ii) in the second stage, this paper estimates the second part of separated steering vector using another least-squares operator and obtains 2D frequencies from the recovered steering vector. The resultant SVSV algorithm does not require spectral search or pairing parameters or singular value decomposition (SVD) of data matrix. Simulation results are presented to validate the performance of the proposed method.

\section{Introduction}

Estimation of two-dimensional (2D) frequencies is a key problem in many areas such as wireless communications, joint frequency and wave-number estimation in array processing, synthetic aperture radar imaging, and nuclear magnetic resonance imaging [1-12]. For example, in the furnace temperature control system of silicon single crystal growth [13], the temperature measurement is affected by the Argon inflation, Crucible turn and rise, Crystal rotation and ascent, and so forth. To suppress these $2 \mathrm{D}$ harmonic interferences, it is necessary to estimate their frequencies accurately, as shown in Figure 1. Numerous methods have been developed to estimate $2 \mathrm{D}$ frequencies. The maximumlikelihood (ML) method [3-5], despite its theoretical optimality, has extremely demanding computational complexity. Some high-resolution techniques, such as autoregressive method [6] and maximum entropy method [7], have limited applications due to spectral peak search in 2D plane. Kay and Nekovei proposed a computationally efficient algorithm in [8], but it is applied to only the single 2D sinusoid case. Both the algebraically coupled matrix pencils (ACMPs) algorithm [9] and 2D ESPRIT method [10] combine two matrix pencils to obtain correct association. The matrix enhancement and matrix pencil (MEMP) method [11] estimates 1D frequencies along each dimension and matches them by maximizing a certain criterion. Unfortunately, the MEMP method does not always provide the correct association. To improve its matching performance, the modified MEMP (MMEMP) method is proposed by Chen et al. [12]. Recently, J. Liu, and X. Liu proposed an eigenvector-based approach, and Haardt et al. developed a higher-order singular value decomposition- (SVD-)based algorithm [14, 15], but both of them require SVD. Besides, they [9-15] suffer performance 


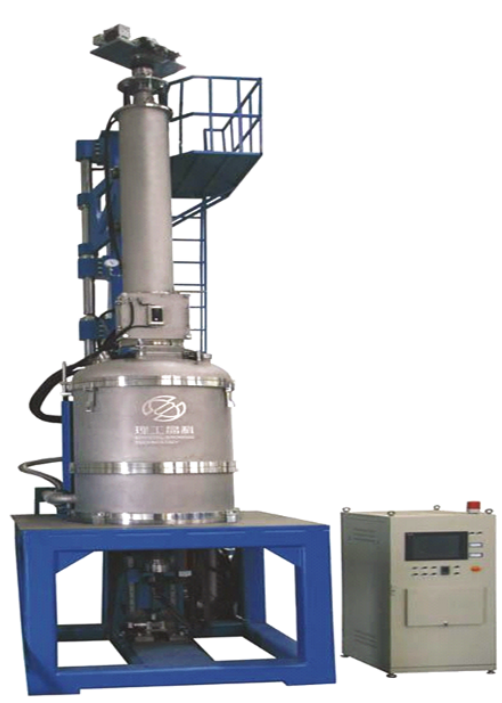

FIgURE 1: TDR-150 CZ crystal furnace. virtual steering vector-based algorithm is developed in Section 3. Simulation results are presented in Section 4. Conclusions are drawn in Section 5.

Throughout this paper, we will vectorize an $m \times n$ matrix $\mathbf{A}$, for example, vec $(\mathbf{A})$, which is the $m n \times 1$ column vector by stacking the row transpose of the matrix $\mathbf{A}$ on top of one another. For example, for the $2 \times 2$ matrix $\mathbf{A}=\left[\begin{array}{ll}a & b \\ c & d\end{array}\right]$,

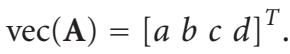

\section{Signal Model}

Consider $K$ superimposed 2D complex-valued sinusoidal signals

$$
x\left(m_{1}, m_{2}\right)=\sum_{k=1}^{K} a_{k} e^{j \phi_{k}} e^{j 2 \pi\left[m_{1} f_{k 1}+m_{2} f_{k 2}\right]}+v\left(m_{1}, m_{2}\right),
$$

where $m_{1}=0,1, \ldots, M_{1}-1, m_{2}=0,1, \ldots, M_{2}-1 . K$ is the number of sinusoidal signals. $a_{k}, \phi_{k}$, and $\left\{f_{k 1}, f_{k 2}\right\}$ are the amplitude, phase, and 2D frequencies of the $k$ th signal, respectively. $v\left(m_{1}, m_{2}\right)$ is the $2 \mathrm{D}$ additive white Gaussian noise (AWGN).

In this paper, we assume that multiple $2 \mathrm{D}$ frequencies may have the same frequency at some dimension, that is, $f_{i 1}=f_{j 1}$ or $f_{i 2}=f_{j 2}$, where $i, j \in\{1,2, \ldots, K\}$, but $f_{i 1}-f_{i 2}$ are different from each other, $i \in\{1,2, \ldots, K\}$. The objective of this paper is to jointly estimate the $2 \mathrm{D}$ frequencies $\left\{f_{k 1}, f_{k 2}\right\}, \quad k=1,2, \ldots, K$, for $K 2 \mathrm{D}$ sinusoidal signals, given the $2 \mathrm{D}$ data $x\left(m_{1}, m_{2}\right)$.

\section{Proposed Algorithm}

3.1. The First Stage of the Proposed SVSV Algorithm. As shown in Figure 2, the original $M_{1} \times M_{2}$-dimensional measurement data can be partitioned into $\prod_{i=1}^{2}\left(M_{i}-L\right)$ snapshots of a virtual rectangular-shaped array with $(L+1)^{2}$ sensors. When the reference sensor (the origin point of rectangular-shaped array) lies on the coordinates $(0,0)$, the single snapshot data of virtual rectangularshaped array consists of $x(0,0), x(0,1), \ldots, x(0, L), \ldots$, $x(L, 0), x(L, 1), \ldots, x(L, L)$, which can be represented in a vector form as

$$
\mathbf{r}(0)=\operatorname{vec}\left(\left[\begin{array}{cccccc}
x(0,0) & x(0,1) & x(0,2) & \cdots & x(0, L-1) & x(0, L) \\
x(1,0) & x(1,1) & x(1,2) & \cdots & x(1, L-1) & x(1, L) \\
x(2,0) & x(2,1) & x(2,2) & \cdots & x(2, L-1) & x(2, L) \\
\vdots & \vdots & \vdots & \cdots & \vdots & \vdots \\
x(L-1,0) & x(L-1,1) & x(L-1,2) & \cdots & x(L-1, L-1) & x(L-1, L) \\
x(L, 0) & x(L, 1) & x(L, 2) & \cdots & x(L, L-1) & x(L, L)
\end{array}\right]\right) \text {. }
$$


Note that $\mathbf{r}(0)$ has the following form:

$$
\mathbf{r}(0)=\mathbf{A s}(0)+\mathbf{n}(0)
$$

$$
\begin{gathered}
\mathbf{A}=\left[\mathbf{a}\left(\gamma_{1}, \phi_{1}\right) \cdots \mathbf{a}\left(\gamma_{k}, \phi_{k}\right) \cdots \mathbf{a}\left(\gamma_{K}, \phi_{K}\right)\right], \\
\gamma_{k}=e^{j 2 \pi f_{k 1}}, \\
\phi_{k}=e^{j 2 \pi f_{k 2}}, \\
\mathbf{n}(0)=\operatorname{vec}\left(\left[\begin{array}{cccccc}
v(0)=\left[\begin{array}{ccccc}
a_{1} e^{j \phi_{1}} \cdots a_{k} e^{j \phi_{k}} & \cdots a_{K} e^{j \phi_{K}}
\end{array}\right]^{T}, \\
v(1,0) & v(0,1) & v(0,2) & \cdots & v(0, L-1) & v(0, L) \\
v(2,0) & v(2,1) & v(2,2) & \cdots & v(2, L-1) & v(2, L) \\
\vdots & \vdots & \vdots & \cdots & \vdots & \vdots \\
v(L-1,0) & v(L-1,1) & v(L-1,2) & \cdots & v(L-1, L-1) & v(L-1, L) \\
v(L, 0) & v(L, 1) & v(L, 2) & \cdots & v(L, L-1) & v(L, L)
\end{array}\right]\right),
\end{gathered}
$$

and, the virtual steering vector $\mathbf{a}\left(\gamma_{k}, \phi_{k}\right)$,

$$
\begin{array}{r}
\mathbf{a}\left(\gamma_{k}, \phi_{k}\right) \\
=\operatorname{vec}\left(\left[\begin{array}{cccccc}
1 & \phi_{k} & \phi_{k}^{2} & \cdots & \phi_{k}^{L-1} & \phi_{k}^{L} \\
\gamma_{k} & \gamma_{k} \phi_{k} & \gamma_{k} \phi_{k}^{2} & \cdots & \gamma_{k} \phi_{k}^{L-1} & \gamma_{k} \phi_{k}^{L} \\
\gamma_{k}^{2} & \gamma_{k}^{2} \phi_{k} & \gamma_{k}^{2} \phi_{k}^{2} & \cdots & \gamma_{k}^{2} \phi_{k}^{L-1} & \gamma_{k}^{2} \phi_{k}^{L} \\
\vdots & \vdots & \vdots & \cdots & \vdots & \vdots \\
\gamma_{k}^{L-1} & \gamma_{k}^{L-1} \phi_{k} & \gamma_{k}^{L-1} \phi_{k}^{2} & \cdots & \gamma_{k}^{L-1} \phi_{k}^{L-1} & \gamma_{k}^{L-1} \phi_{k}^{L} \\
\gamma_{k}^{L} & \gamma_{k}^{L} \phi_{k} & \gamma_{k}^{L} \phi_{k}^{2} & \cdots & \gamma_{k}^{L} \phi_{k}^{L-1} & \gamma_{k}^{L} \phi_{k}^{L}
\end{array}\right]\right) .
\end{array}
$$

When the reference sensor "moves" from coordinates $(0,0)$ to $\left(0, M_{2}-L-1\right)$, from $(1,0)$ to $\left(1, M_{2}-L-1\right)$, from $(2,0)$ to $\left(2, M_{2}-L-1\right), \ldots$, from $\left(M_{1}-L-1,0\right)$ to $\left(M_{1}-L-1, M_{2}-\right.$ $L-1)$ with other sensors of virtual rectangular-shaped array, $\prod_{i=1}^{2} M_{i}-l$ virtual snapshots are available and can be written in the following form:

$$
\mathbf{r}(t)=\mathbf{A s}(t)+\mathbf{n}(t), \quad t=0,1, \ldots, \prod_{i=1}^{2}\left(M_{i}-L\right)-1
$$

Let $q=t \bmod \left(M_{2}-L\right)$, where "mod" stands for the remainder operator and $q$ is the remainder. Thus, $p=(t-q)$ /
$\left(M_{2}-L\right)$ is the corresponding quotient. Therefore, $\mathbf{s}(t)$ has the following form:

$$
\begin{gathered}
\mathbf{s}(t)=\left[a_{1} e^{j \phi_{1}} e^{j 2 \pi\left(f_{11} p+f_{12} q\right)} \cdots a_{k} e^{j \phi_{k}} e^{j 2 \pi\left(f_{k 1} p+f_{k 2} q\right)}\right. \\
\left.\cdots \times a_{K} e^{j \phi_{K}} e^{j 2 \pi\left(f_{K 1} p+f_{K 2} q\right)}\right]^{T} \\
t=0,1, \ldots, \prod_{i=1}^{2}\left(M_{i}-L\right)-1
\end{gathered}
$$

Therefore, $\mathbf{r}(t), t=0,1, \ldots, \prod_{i=1}^{2}\left(M_{i}-L\right)-1$, can be combined to form the following data matrix:

$$
\begin{aligned}
\mathbf{R}_{u}= & {\left[\mathbf{r}(0) \cdots \mathbf{r}\left(\left(M_{1}-L\right) \times\left(M_{2}-L\right)-1\right)\right] } \\
= & \mathbf{A}\left[\mathbf{s}(0) \cdots \mathbf{s}\left(\left(M_{1}-L\right) \times\left(M_{2}-L\right)-1\right)\right] \\
& +\left[\mathbf{n}(0) \cdots \mathbf{n}\left(\left(M_{1}-L\right) \times\left(M_{2}-L\right)-1\right)\right] .
\end{aligned}
$$

Considering that spatial smoothing can improve the estimation accuracy [20], we use $\mathbf{R}=\left[\begin{array}{ll}\mathbf{R}_{u} & J \mathbf{R}_{u}^{*}\end{array}\right]$ as the virtual array data, where $\mathbf{J}$ is an antidiagonal matrix in which all nonzero elements are equal to one.

In the conventional case, multiple $2 \mathrm{D}$ frequencies have the same frequency at some dimension, that is, $f_{i 1}=f_{j 1}$ or $f_{i 2}=f_{j 2}$, where $i, j \in\{1,2, \ldots, K\}$. Some existing methods estimate $2 \mathrm{D}$ frequencies along each dimension and associate the two sets of separately estimated one-dimensional (1D) frequencies into a set of $2 \mathrm{D}$ frequencies. Therefore, incorrect match is often encountered in this case, which results in severe performance degradation.

To overcome this difficulty, we define

$$
z_{k}=\frac{\phi_{k}}{\gamma_{k}}=\frac{e^{j 2 \pi f_{k 2}}}{e^{j 2 \pi f_{k 1}}}=e^{j 2 \pi\left(f_{k 2}-f_{k 1}\right)}, k=1, \ldots, K .
$$




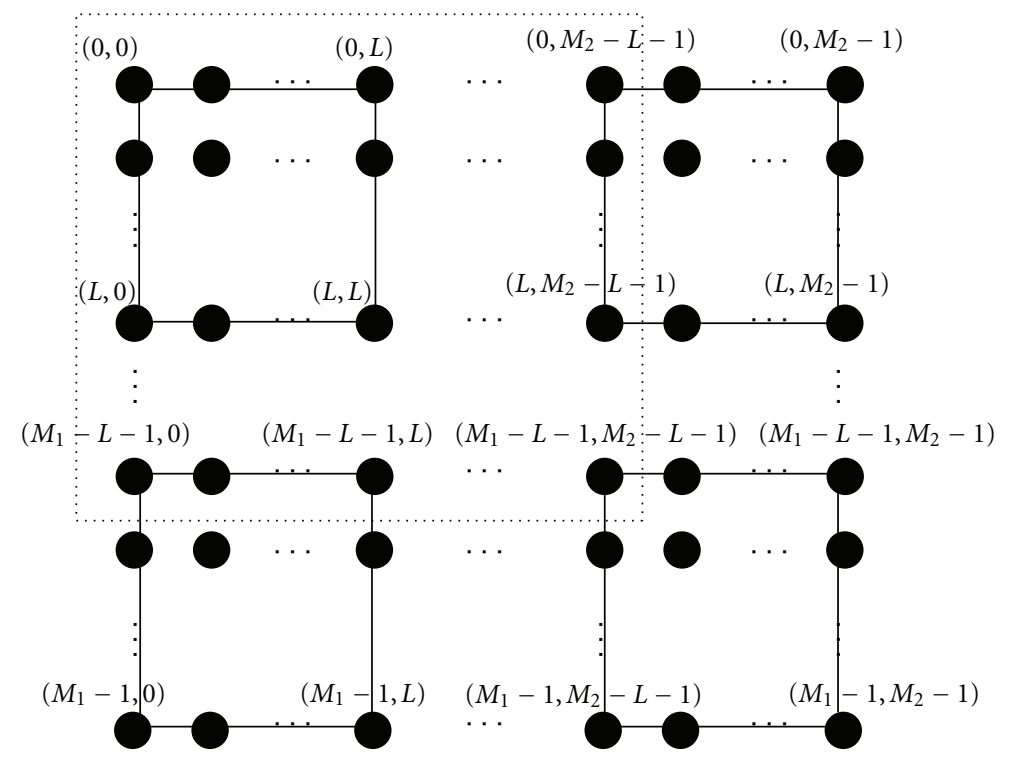

FIgURE 2: The formation of virtual array data from $x\left(m_{1}, m_{2}\right)$. [21]

Since $\left|z_{k}\right|=1, z_{k}$ can be represented in another form as

$$
z_{k}=e^{j \theta_{k}}, \quad \theta_{k} \in[-\pi, \pi], k=1, \ldots, K .
$$

Although $e^{j \theta_{k}}=e^{j 2 \pi\left(f_{k 2}-f_{k 1}\right)}, \theta_{k} \in[-\pi, \pi]$ may not equal $2 \pi\left(f_{k 2}-f_{k 1}\right)$ due to $2 \pi f_{k 1}, 2 \pi f_{k 2} \in[-\pi, \pi]$ and thus $2 \pi\left(f_{k 2}-\right.$ $\left.f_{k 1}\right)$ may exceed $[-\pi, \pi]$, that is, $2 \pi\left(f_{k 2}-f_{k 1}\right)$ cannot be estimated from $\theta_{k}$ directly, but $e^{j 2 \pi\left(f_{k 2}-f_{k 1}\right)}$ can be estimated from $e^{j \theta_{k}}$ by estimating $\theta_{k}$ in $[-\pi, \pi]$.

Based on (9)-(10), $\phi_{k}$ can be represented as $\gamma_{k} z_{k}$. Therefore, the steering vector $\mathbf{a}\left(\gamma_{k}, \phi_{k}\right)$ in (5) can be written in another form as follows:

$$
\begin{aligned}
& \mathbf{a}\left(\gamma_{k}, z_{k}\right) \\
& =\operatorname{vec}\left(\left[\begin{array}{cccccc}
1 & \gamma_{k} z_{k} & \gamma_{k}^{2} z_{k}^{2} & \cdots & \gamma_{k}^{L-1} z_{k}^{L-1} & \gamma_{k}^{L} z_{k}^{L} \\
\gamma_{k} & \gamma_{k}^{2} z_{k} & \gamma_{k}^{3} z_{k}^{2} & \cdots & \gamma_{k}^{L} z_{k}^{L-1} & \gamma_{k}^{L+1} z_{k}^{L} \\
\gamma_{k}^{2} & \gamma_{k}^{3} z_{k} & \gamma_{k}^{4} z_{k}^{2} & \cdots & \gamma_{k}^{L+1} z_{k}^{L-1} & \gamma_{k}^{L+2} z_{k}^{L} \\
\vdots & \vdots & \vdots & \cdots & \vdots & \vdots \\
\gamma_{k}^{L-1} & \gamma_{k}^{L} z_{k} & \gamma_{k}^{L+1} z_{k}^{2} & \cdots & \gamma_{k}^{2 L-2} z_{k}^{L-1} & \gamma_{k}^{2 L-1} z_{k}^{L} \\
\gamma_{k}^{L} & \gamma_{k}^{L+1} z_{k} & \gamma_{k}^{L+2} z_{k}^{2} & \cdots & \gamma_{k}^{2 L-1} z_{k}^{L-1} & \gamma_{k}^{2 L} z_{k}^{L}
\end{array}\right]\right) .
\end{aligned}
$$

Introduce the following partition of $\mathbf{R}$ :

$$
\mathbf{R}=\left[\begin{array}{l}
\mathbf{R}_{1} \\
\mathbf{R}_{2}
\end{array}\right],
$$

where $\mathbf{R}_{1}$ and $\mathbf{R}_{2}$ are the first $K$ rows and the rest of matrix $\mathbf{R}$, respectively.

Define the propagator

$$
\mathbf{P}=\left(\mathbf{R}_{1} \mathbf{R}_{1}^{H}\right)^{-1} \mathbf{R}_{1} \mathbf{R}_{2}^{H},
$$

using least-squares operator, which satisfies with $\mathbf{P}=$ $\min _{\mathbf{P}}\left\|\mathbf{R}_{2}-\mathbf{P}^{H} \mathbf{R}_{1}\right\|^{2}$. Based on the conventional propagator method $[18,19,22],\left(\gamma_{k}, z_{k}\right)$ satisfies

$$
\left[\begin{array}{c}
\mathbf{P} \\
-\mathbf{I}_{(L+1)^{2}-K}
\end{array}\right]^{H} \mathbf{a}\left(\gamma_{k}, z_{k}\right)=\mathbf{0}, \quad k=1,2, \ldots, K,
$$

or

$$
\begin{gathered}
\mathbf{g}\left(\gamma_{k}, z_{k}\right)=\mathbf{a}^{H}\left(\gamma_{k}, z_{k}\right)\left[\begin{array}{c}
\mathbf{P} \\
-\mathbf{I}_{(L+1)^{2}-K}
\end{array}\right]\left[\begin{array}{c}
\mathbf{P} \\
-\mathbf{I}_{(L+1)^{2}-K}
\end{array}\right]^{H} \\
\times \mathbf{a}\left(\gamma_{k}, z_{k}\right)=0, \quad k=1,2, \ldots, K .
\end{gathered}
$$

Based on (11), the steering vector $\mathbf{a}\left(\gamma_{k}, z_{k}\right)$ of virtual array can be represented in separated two parts as

$$
\mathbf{a}\left(\gamma_{k}, z_{k}\right)=\mathbf{a}_{1}\left(z_{k}\right) \mathbf{a}_{2}\left(\gamma_{k}\right),
$$

where $(2 L+1)$-dimensional vector

$$
\mathbf{a}_{2}\left(\gamma_{k}\right)=\left[\begin{array}{llllll}
1 & \gamma_{k} & \gamma_{k}^{2} & \gamma_{k}^{3} \cdots & \gamma_{k}^{2 L-1} & \gamma_{k}^{2 L}
\end{array}\right]^{T},
$$

and in, $(L+1)^{2} \times(2 L+1)$-dimensional matrix $\mathbf{a}_{1}\left(z_{k}\right)$,

$$
\mathbf{a}_{\mathbf{1}}\left(\mathbf{z}_{\mathbf{k}}\right)=\left[\begin{array}{cc}
\mathrm{C} & \mathbf{0}_{(\mathrm{L}+\mathbf{1}) \times \mathrm{L}} \\
\mathbf{0}_{(\mathrm{L}+1) \times 1} \mathrm{C} & \mathbf{0}_{(\mathrm{L}+1) \times(\mathrm{L}-1)} \\
\boldsymbol{0}_{(\mathrm{L}+1) \times 2} \mathrm{C} & \mathbf{0}_{(\mathrm{L}+1) \times(\mathrm{L}-\mathbf{2})} \\
\vdots \\
\mathbf{0}_{(\mathrm{L}+\mathbf{1}) \times(\mathrm{L}-\mathbf{1})} \mathrm{C} & \mathbf{0}_{(\mathrm{L}+\mathbf{1}) \times \mathbf{1}} \\
\mathbf{0}_{(\mathrm{L}+\mathbf{1}) \times \mathrm{L}} \mathrm{C}
\end{array}\right],
$$


in which

$$
\mathbf{C}=\operatorname{diag}\left\{1, z_{k}, z_{k}^{2}, \ldots, z_{k}^{L}\right\}
$$

Inserting (16) into (15), we have

$$
\begin{aligned}
g\left(\gamma_{k}, z_{k}\right) & \\
= & \mathbf{a}^{H}\left(\gamma_{k}, z_{k}\right)\left[\begin{array}{c}
\mathbf{P} \\
-\mathbf{I}_{(L+1)^{2}-K}
\end{array}\right]\left[\begin{array}{c}
\mathbf{P} \\
-\mathbf{I}_{(L+1)^{2}-K}
\end{array}\right]^{H} \mathbf{a}\left(\gamma_{k}, z_{k}\right) \\
= & \mathbf{a}_{2}^{H}\left(\gamma_{k}\right) \mathbf{a}_{1}^{H}\left(z_{k}\right)\left[\begin{array}{c}
\mathbf{P} \\
-\mathbf{I}_{(L+1)^{2}-K}
\end{array}\right]\left[\begin{array}{c}
\mathbf{P} \\
-\mathbf{I}_{(L+1)^{2}-K}
\end{array}\right]^{H} \\
& \times \mathbf{a}_{1}\left(z_{k}\right) \mathbf{a}_{2}\left(\gamma_{k}\right) \\
= & \mathbf{a}_{2}^{H}\left(\gamma_{k}\right) \mathbf{D}\left(z_{k}\right) \mathbf{a}_{2}\left(\gamma_{k}\right) \\
= & 0
\end{aligned}
$$

in the ideal case $[18,19]$, where

$$
\mathbf{D}(z)=\mathbf{a}_{1}^{H}(z)\left[\begin{array}{c}
\mathbf{P} \\
-\mathbf{I}_{(L+1)^{2}-K}
\end{array}\right]\left[\begin{array}{c}
\mathbf{P} \\
-\mathbf{I}_{(L+1)^{2}-K}
\end{array}\right]^{H} \mathbf{a}_{1}(z) .
$$

Note that if $2 L+1 \leq(L+1)^{2}-K$ then $D(z)$ is, in general, full rank because the column rank of $\left[\begin{array}{c}\mathbf{P} \\ -\mathbf{I}_{(L+1)^{2}-K}\end{array}\right]$ is $(L+1)^{2}-$ $K$. Equation (20) may hold true if and only if matrix $\mathbf{D}(z)$ drops rank (i.e., $2 L)$, or equivalently, when the polynomial of $z=z_{k}$ (see (21) for details),

$$
\operatorname{det}\{\mathbf{D}(z)\}=0
$$

which is the rank-reduction propagator method (RRPM) [23]. From the roots $z_{k}=e^{j \theta_{k}}$ of (22) [20], it is easy to find $\theta_{k}, k=1,2, \ldots, K$. Equations (15) and (20) imply that, substituting the estimated $\widehat{z}_{k}$ into $\mathbf{a}_{1}\left(z_{k}\right)$ in (20), then we find the minima of the following function:

$$
\hat{\gamma}_{k}=\min _{\gamma} \mathbf{a}_{2}^{H}(\gamma) \mathbf{D}\left(\hat{z}_{k}\right) \mathbf{a}_{2}(\gamma)
$$

the minima of which indicates the estimation $\hat{\gamma}_{k}$. In fact, Equation (20) implies that $\mathbf{a}_{2}\left(\gamma_{k}\right)$ is just the unique eigenvector corresponding to the smallest eigenvalue (zero) of $\mathbf{D}\left(\hat{z}_{k}\right)$ in the ideal case. In other words,

$$
\left[\begin{array}{c}
\mathbf{P} \\
-\mathbf{I}_{(L+1)^{2}-K}
\end{array}\right]^{H} \mathbf{a}_{1}\left(z_{k}\right) \mathbf{a}_{2}\left(\gamma_{k}\right)=\mathbf{0} .
$$

3.2. The Second Stage of the Proposed SVSV Algorithm. Define

$$
\mathbf{D}_{2}=\left[\begin{array}{ll}
\mathbf{D}_{21} & \mathbf{D}_{22}
\end{array}\right]=\left[\begin{array}{c}
\mathbf{P} \\
-\mathbf{I}_{(L+1)^{2}-K}
\end{array}\right]^{H} \mathbf{a}_{1}\left(\hat{z}_{k}\right),
$$

where $\mathbf{D}_{21}$ and $\mathbf{D}_{22}$ are the first columns and last $2 L$ column of $\mathbf{D}_{2}$, respectively.

From (25), we can see that

$$
\begin{aligned}
& {\left[\begin{array}{ll}
\mathbf{D}_{21} & \mathbf{D}_{22}
\end{array}\right] a_{2}\left(\gamma_{k}\right)}
\end{aligned}
$$

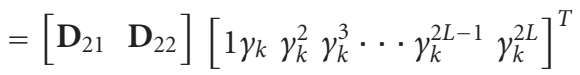

$$
\begin{aligned}
& =\mathbf{D}_{21}+\mathbf{D}_{22}\left[\begin{array}{llllll}
\gamma_{k} & \gamma_{k}^{2} & \gamma_{k}^{3} & \cdots & \gamma_{k}^{2 L-1} & \gamma_{k}^{2 L}
\end{array}\right]^{T} \\
& =\mathbf{0}
\end{aligned}
$$

or

$$
\mathbf{D}_{22}\left[\begin{array}{llllll}
\gamma_{k} & \gamma_{k}^{2} & \gamma_{k}^{3} & \cdots & \gamma_{k}^{2 L-1} & \gamma_{k}^{2 L}
\end{array}\right]^{T}=-\mathbf{D}_{21}
$$

which implies that $\left[\begin{array}{llllll}\gamma_{k} & \gamma_{k}^{2} & \gamma_{k}^{3} \cdots \gamma_{k}^{2 L-1} & \gamma_{k}^{2 L}\end{array}\right]^{T}$ can be obtained using another least-square operation

$$
\left[\begin{array}{lllll}
\gamma_{k} & \gamma_{k}^{2} & \gamma_{k}^{3} & \cdots & \gamma_{k}^{2 L-1} \\
\gamma_{k}^{2 L}
\end{array}\right]^{T}=-\left(\mathbf{D}_{22}^{H} \mathbf{D}_{22}\right)^{-1} \mathbf{D}_{22}^{H} \mathbf{D}_{21} .
$$

3.3. Description of the Proposed SVSV Algorithm. Based on the above analyses, the proposed SVSV algorithm for 2D frequency estimation can be described as follows:

Step 1. Construct data matrix $\mathbf{R}$ using (2)-(8).

Step 2. The partition of $\mathbf{R}$ yields $\mathbf{R}_{1}$ and $\mathbf{R}_{2}$ using (12), from which the propagator $\mathbf{P}=\left(\mathbf{R}_{1} \mathbf{R}_{1}^{H}\right)^{-1} \mathbf{R}_{1} \mathbf{R}_{2}^{H}$ is obtained.

Step 3. Obtain $z_{k}$ from the roots of polynomial $\mathbf{D}(z)$ in (22).

Step 4. $\mathbf{a}_{2}\left(\hat{\gamma}_{k}\right)$ is easily constructed from the least-square solution of (28).

Step 5. Obtain $\hat{\gamma}_{k}$ from $\mathbf{a}_{2}\left(\hat{\gamma}_{k}\right)$ according to the following equation:

$$
\gamma_{k}=\frac{1}{2 L} \sum_{i=1}^{2 L} \frac{\mathbf{a}_{2}\left(\hat{\gamma}_{k}\right)[i+1]}{\mathbf{a}_{2}\left(\hat{\gamma}_{k}\right)[i]},
$$

where $\mathbf{a}_{2}\left(\hat{\gamma}_{k}\right)[i]$ denotes the $i$ th element of $\mathbf{a}_{2}\left(\hat{\gamma}_{k}\right)$.

Step 6. The estimation $\left\{\hat{f}_{k 1}, \hat{f}_{k 2}\right\}$ can be given as follows:

$$
\begin{gathered}
f_{k 1}=\frac{1}{2 \pi} \angle \hat{\gamma}_{k}, \\
f_{k 2}=\frac{1}{2 \pi} \angle\left(\hat{\gamma}_{k} \hat{z}_{k}\right) .
\end{gathered}
$$

3.4. Discussion. In this section, we discuss the proposed algorithm in three ways, that is, computational complexity, selection of $L$, and capacity for estimating 2D frequencies when multiple $2 \mathrm{D}$ frequencies have the same frequency at some dimension. 
Computational Complexity. Regarding the computational complexity, we consider the major part, namely, multiplications and SVD. The eigenvector method addressed [14] requires $O\left\{M_{1}^{3} M_{2}^{3}\right\}$, and the MMEMP algorithm developed in [12] needs $O\left\{L^{2} M_{1}^{2} M_{2}^{2}\right\}$. However, the proposed SVSV algorithm without SVD requires $O\left\{M_{1} M_{2} L\right\}$, being less than those of the eigenvector method and MMEMP algorithm.

About Selection of $L$. the original $M_{1} \times M_{2}$ measurement data can be partitioned into $\prod_{i=1}^{2}\left(M_{i}-L\right)$ snapshots of a virtual rectangular-shaped array with $(L+1)^{2}$ sensors. In the array signal processing field, the increase of sensors (large array aperture) can improve the direction-of-arrival (DOA) estimation accuracy under the given snapshots, and so does the increase of snapshots under the given sensors [16-18]. However, for the 2D frequencies estimation problem, the rise of virtual sensors is contradictory to the increase of the virtual snapshots, given $M_{1} \times M_{2}$ measurement data (see the relationship between $(L+1)^{2}$ and $\prod_{i=1}^{2}\left(M_{i}-L\right)$. In this case, more virtual sensors (i.e., $(L+1)^{2}$ or larger array aperture) means that less snapshots $\left(\prod_{i=1}^{2}\left(M_{i}-L\right)\right)$ are available, which causes low estimation accuracy of $\mathbf{R}$ or $\mathbf{P}=\left(\mathbf{R}_{1} \mathbf{R}_{1}^{H}\right)^{-1} \mathbf{R}_{1} \mathbf{R}_{2}^{H}$. On the other hand, more virtual snapshots $\left(\prod_{i=1}^{2}\left(M_{i}-L\right)\right)$ means that less virtual sensors are available, which causes small array aperture. Therefore, the selection of $L$ should be evaluated from available virtual snapshots and sensors. From the second experiment in Section 4, we can see that both too large $L$ and small $L$ cause low estimation accuracy, which is in agreement with the above analyses.

Capacity for Estimating 2D Frequencies When Multiple 2D Frequencies Have the Same Frequency at some Dimension. In the conventional case, multiple 2D frequencies have the same frequency at some dimension, that is, $f_{i 1}=f_{j 1}$ or $f_{i 2}=f_{j 2}$, where $i, j \in\{1,2, \ldots, K\}$. Although $f_{i 1}=f_{j 1}$ or $f_{i 2}=f_{j 2}$, $f_{i 1}-f_{i 2}$ is different from each other, $i \in\{1,2, \ldots, K\} . z_{k}$ are determined from the roots of polynomial $\mathbf{D}(z)$ in (22) and the related $\mathbf{a}_{2}\left(\gamma_{k}\right)$ (i.e., the unique eigenvector corresponding to the smallest eigenvalue (zero) of $\mathbf{D}\left(\hat{z}_{k}\right)$ ) is obtained using least-squares operator. Therefore, the proposed SVSV algorithm can implement efficiently especially when multiple 2D frequencies have the same frequency at some dimension and avoid pairing parameters. However, when $f_{i 1}-f_{i 2}$ are equals to each other, $i \in\{1,2, \ldots, K\}$, polynomial $\mathbf{D}(z)$ in (22) has several equivalent roots, $z_{1}=z_{2}=\cdots=z_{K}$. In this case, $\mathbf{a}_{2}\left(\gamma_{k}\right), k \in\{1,2, \ldots, K\}$, is no longer the unique eigenvector, but one of several eigenvectors corresponding to the smallest eigenvalue (zero) of $\mathbf{D}\left(\hat{z}_{k}\right)$. Therefore, unequal $f_{i 1}-f_{i 2}, i \in\{1,2, \ldots, K\}$, are required to ensure unique estimation for the proposed algorithm.

\section{Simulation Results}

In the simulation section, we compare the SVSV algorithm with the MMEMP algorithm [12] and the eigenvector method [13] to assess the estimation performance of the proposed algorithm.

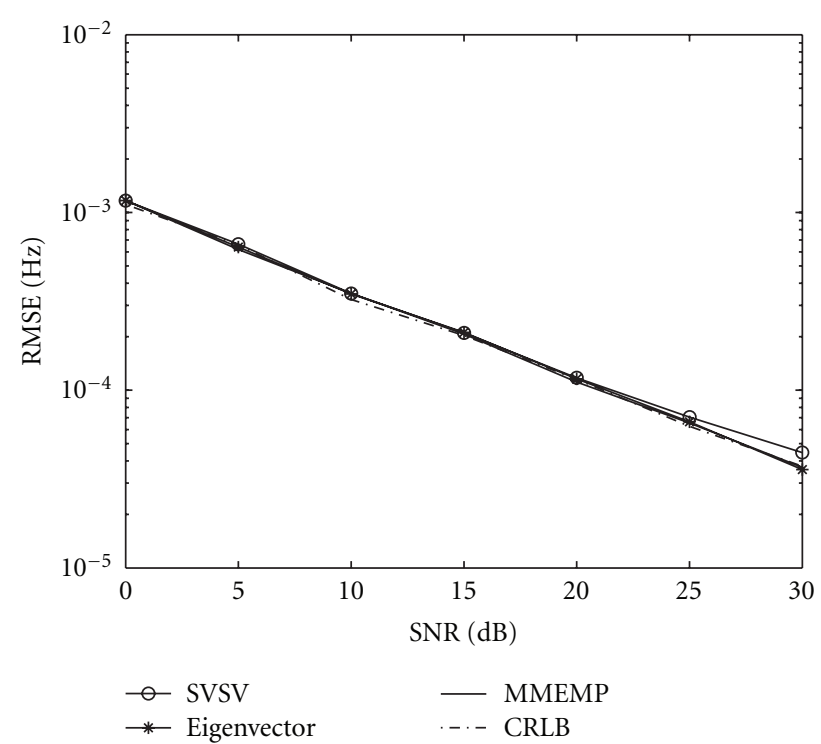

Figure 3: RMSE of the estimated frequency $f_{11}$ versus SNR.

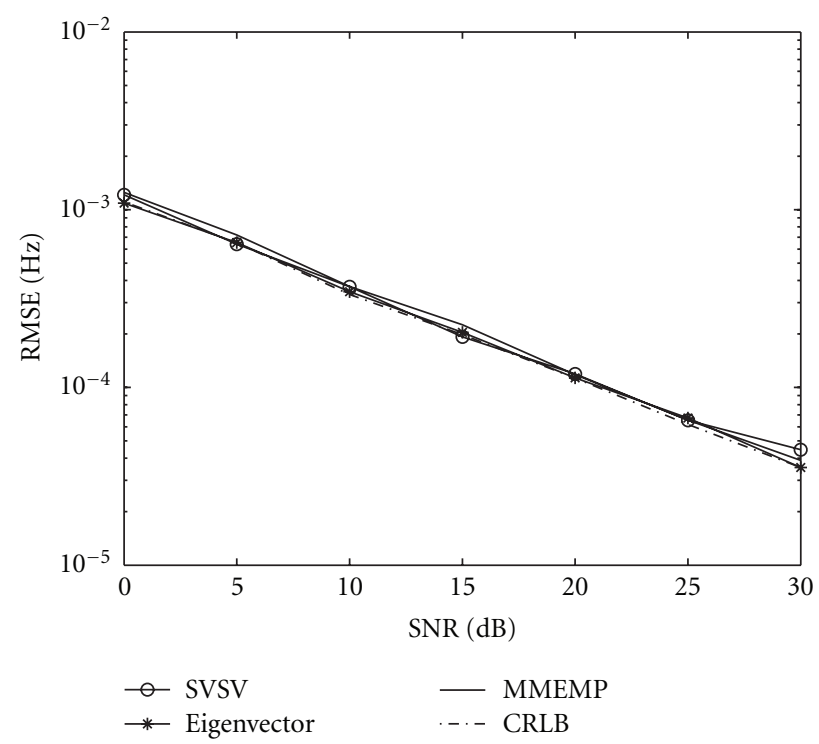

FIGURE 4: RMSE of the estimated frequency $f_{12}$ versus SNR.

In the first experiment, $20 \times 20$ data samples with $f_{11}=$ $0.20837, f_{12}=0.09943, f_{21}=0.15077, f_{22}=0.25789, f_{31}=$ 0.10231 , and $f_{32}=0.30987$ are used. The effect of signalto-noise ratio (SNR) on the performance of the proposed SVSV algorithm is investigated. The SNR varies from $0 \mathrm{~dB}$ to $30 \mathrm{~dB}$. We use the root mean square error (RMSE) of the estimated frequencies as the performance evaluation. Figures 3, 4, 5, 6, 7, and 8, respectively, show the RMSE of estimated six frequencies from 500 independent runs using these algorithms: the proposed algorithm $(L=6)$, the MMEMP algorithm $(K=L=6$, enhancement matrix [12, Equation(5.7)] for all three experiments, and the eigenvector method $\left(K_{1}=K_{2}=6\right.$, enhancement matrix 


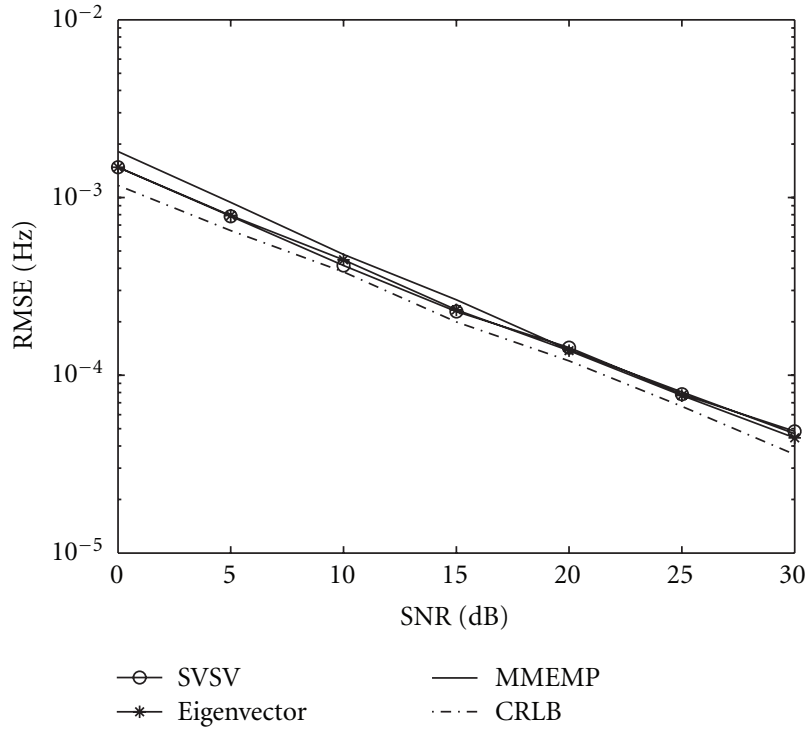

Figure 5: RMSE of the estimated frequency $f_{21}$ versus SNR.

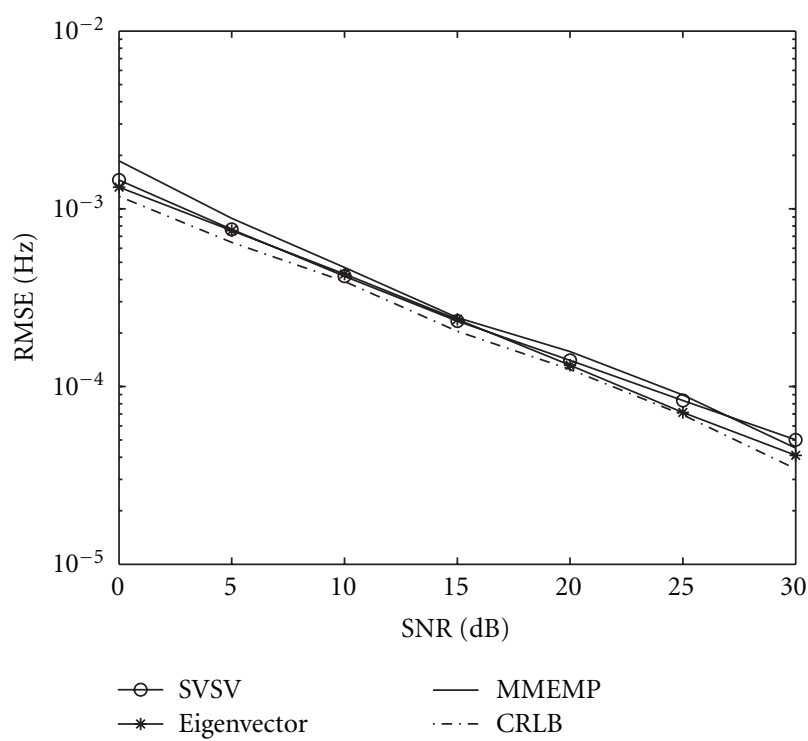

Figure 6: RMSE of the estimated frequency $f_{22}$ versus SNR.

[14]). In addition, the related CRLB on the variance of the estimated parameters is obtained from the inverse of the Fisher information matrix by averaging 500 computations [23]. From these figures, we can see that the eigenvector method and the proposed algorithm slightly outperform the MMEMP method in the estimation accuracy under different SNRs, and their RMSE approach to the CRLB.

In the second experiment, $20 \times 20$ data samples with $f_{11}=$ 0.20837, $f_{12}=0.09943, f_{21}=0.15077, f_{22}=0.25789, f_{31}=$ 0.10231 , and $f_{32}=0.30987$ are used. The effect $L$ on the performance of the proposed SVSV algorithm is investigated. The SNR is set to $10 \mathrm{~dB}$. Figure 9 shows the RMSE of the estimated frequencies for 500 independent runs using the

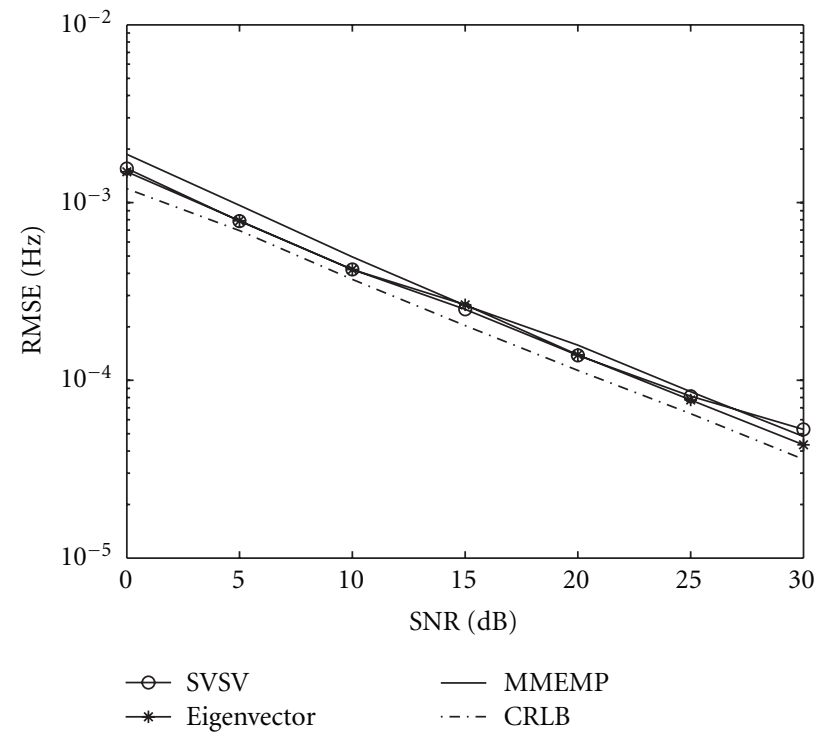

Figure 7: RMSE of the estimated frequency $f_{31}$ versus SNR.

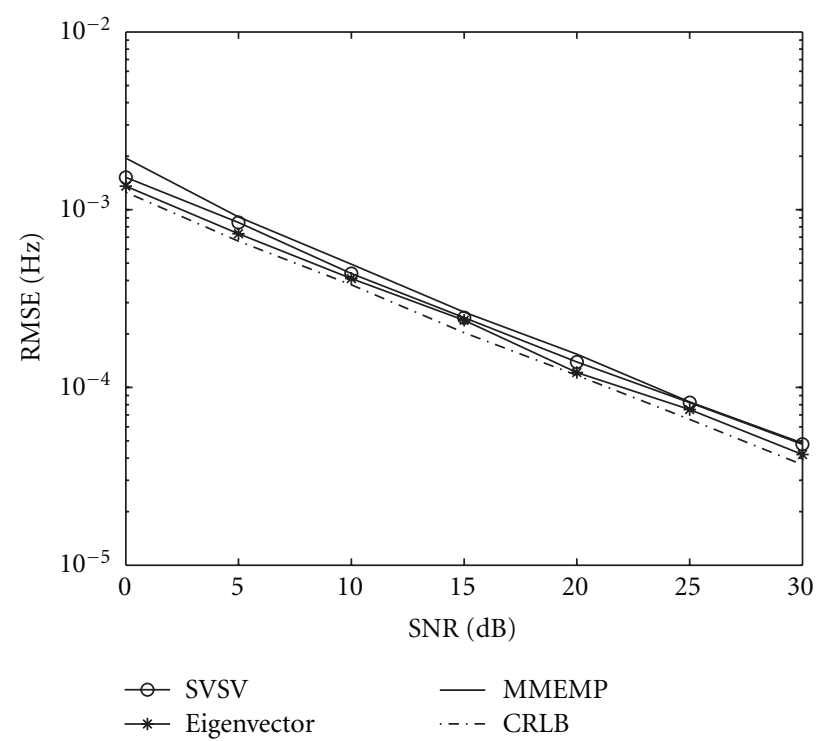

Figure 8: RMSE of the estimated frequency $f_{32}$ versus SNR.

proposed SVSV algorithm with different $L$. From it, we can see that for the simulation data the proposed algorithm has better estimation performance when $L=6$.

In the third experiment, $20 \times 20$ data samples with $f_{11}=$ $0.20837, f_{12}=0.09943, f_{21}=0.10231, f_{22}=0.25789, f_{31}=$ 0.10231 , and $\left.f_{32}=0.09943\right)$ are used. The ability of the proposed SVSV algorithm to deal with the same frequencies at some dimension is investigated. The SNR varies from $0 \mathrm{~dB}$ to $30 \mathrm{~dB}$. Figures $10,11,12,13,14$, and 15, respectively, show the RMSE of the estimated six frequencies for 500 independent runs using these algorithms: the proposed algorithm $(L=6)$, the MMEMP algorithm $(K=L=$ 6, enhancement matrix[12, Equations (5-7)] for all three 


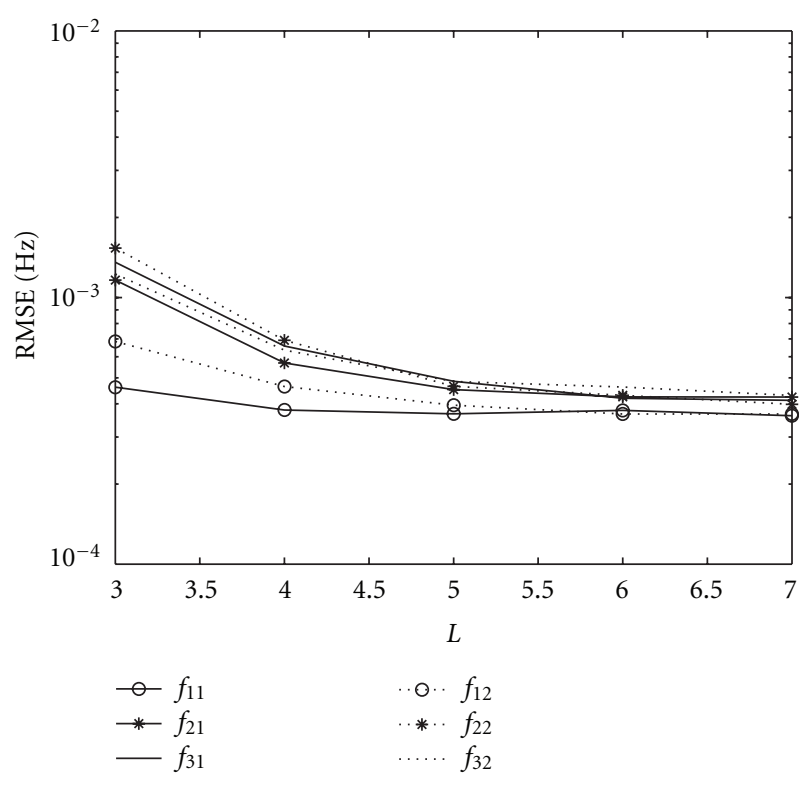

FIGURE 9: RMSE of the estimated frequencies versus $L$.

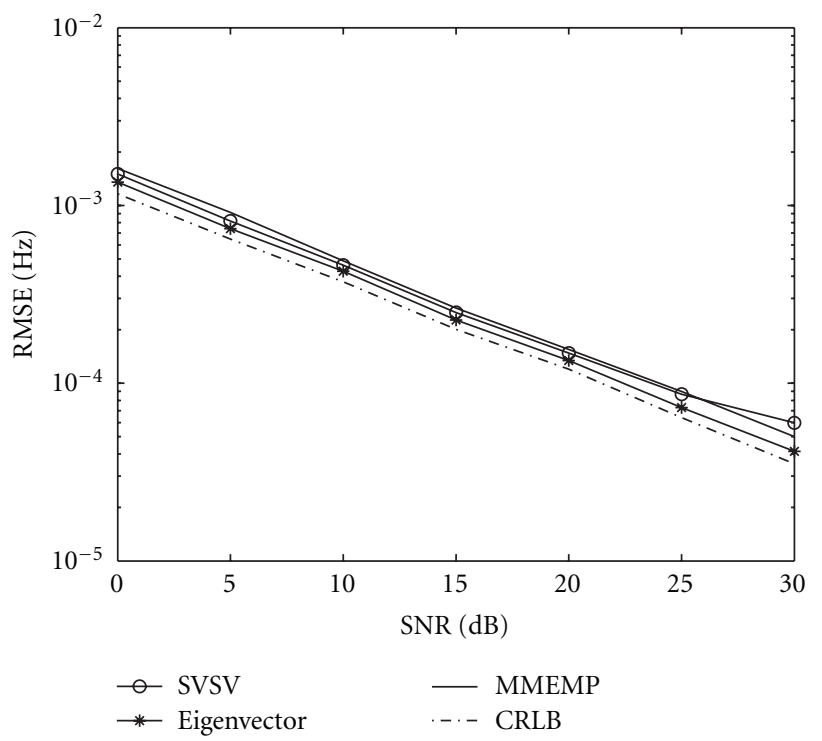

FIGURE 10: RMSE of the estimated frequency $f_{11}$ versus SNR.

experiments, and the eigenvector method $\left(K_{1}=K_{2}=\right.$ 6 , enhancement matrix [14]). The eigenvector algorithm obtains the eigenvectors being corresponding to eigenvalues $e^{j 2 \pi f_{11}}, e^{j 2 \pi f_{21}}$, and $e^{j 2 \pi f_{31}}$, and thus has poor estimation performance due to $f_{21}=f_{31}$ at the first dimension. The similar reason holds for the MMEMP method. From Figures 13 and 15, we can see that the eigenvector algorithm and MMEMP method have low estimation accuracy for $f_{22}$ and $f_{32}$ due to $f_{21}=f_{31}$. However, the proposed SVSV algorithm performs well in distinguishing the three signals.

In the fourth experiment, $20 \times 20$ data samples with $f_{11}=0.20837, f_{12}=0.09943, f_{21}=0.10231, f_{22}=$ $0.25789, f_{31}=0.10231$, and $f_{32}=0.09943$ are used.

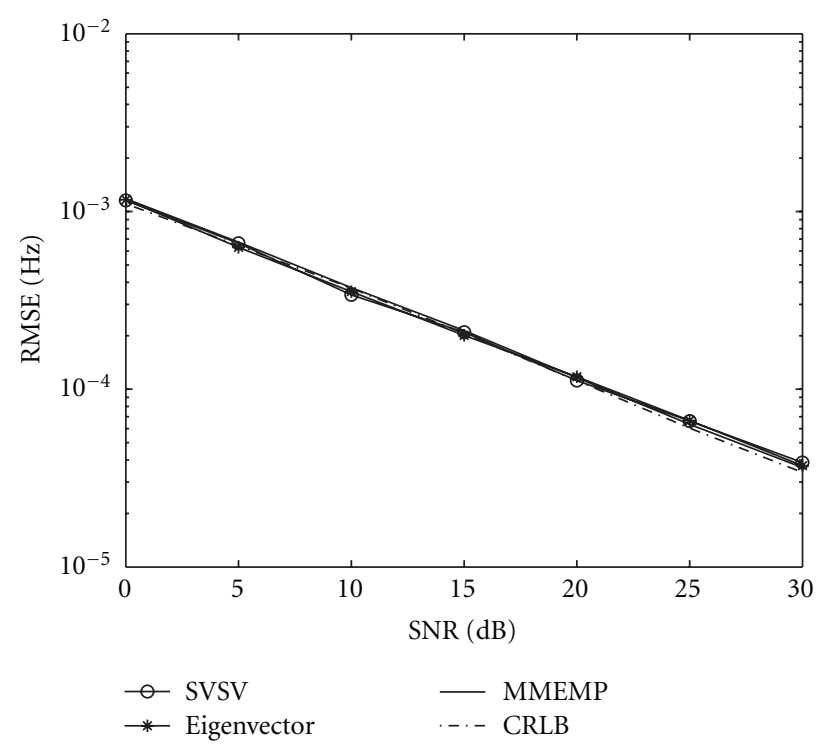

FIGURE 11: RMSE of the estimated frequency $f_{12}$ versus SNR.

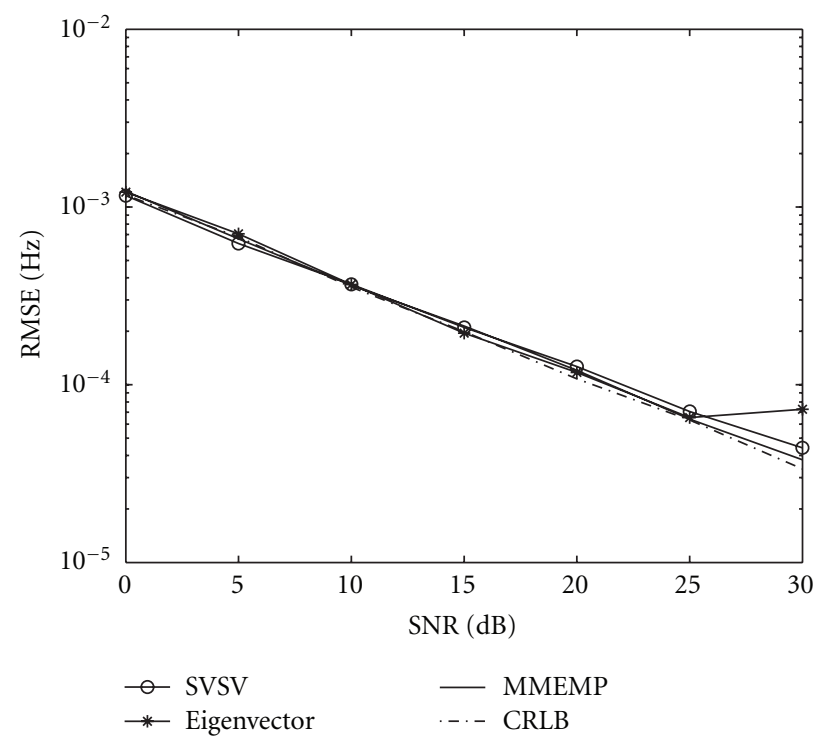

FIGURE 12: RMSE of the estimated frequency $f_{21}$ versus SNR.

The SNR is set to $10 \mathrm{~dB}$. Figures 16,17 , and 18 show $2 \mathrm{D}$ plot of the estimated frequencies for 500 independent runs using the proposed algorithm, the MMEMP algorithm, and the eigenvector method, respectively. From these figures, we can see that the MMEMP and eigenvector methods implement poorly when multiple $2 \mathrm{D}$ frequencies have the same frequency at some dimension, that is, $f_{21}=f_{31}$. However, the proposed algorithm performs well because $f_{11}-f_{12}, f_{21}-f_{22}$, and $f_{31}-f_{32}$ are different from each other.

In the fifth experiment, $20 \times 20$ data samples with two signals are used, in which the first one is with $2 \mathrm{D}$ frequencies $\left.f_{11}=0.20837, f_{12}=0.09943\right)$, and the second one is with $f_{21}=0.15077$. The SNR is set as $10 \mathrm{~dB}$. When $f_{22}$ varies from 


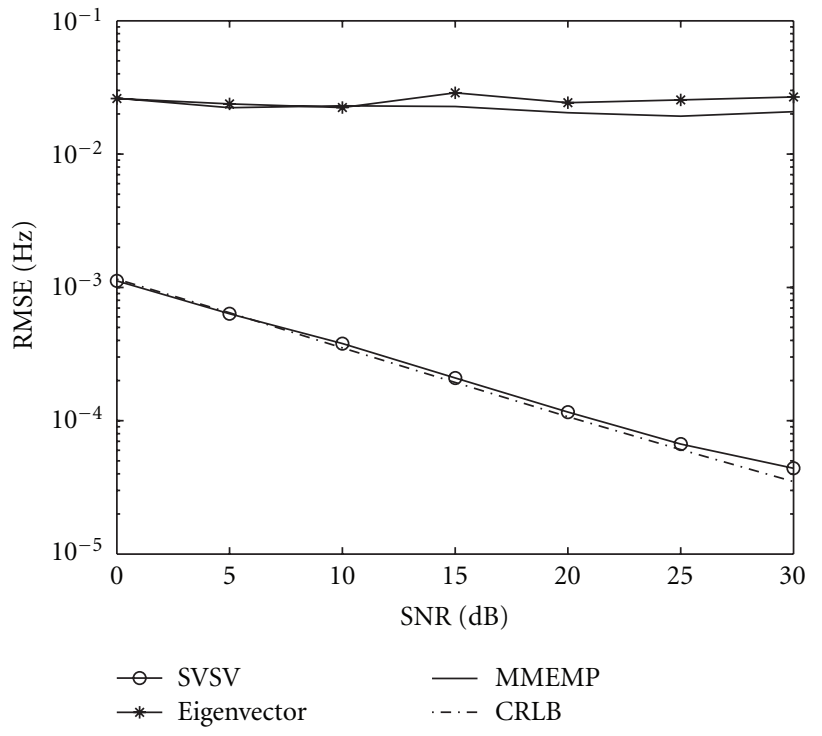

FIGURE 13: RMSE of the estimated frequency $f_{22}$ versus SNR.

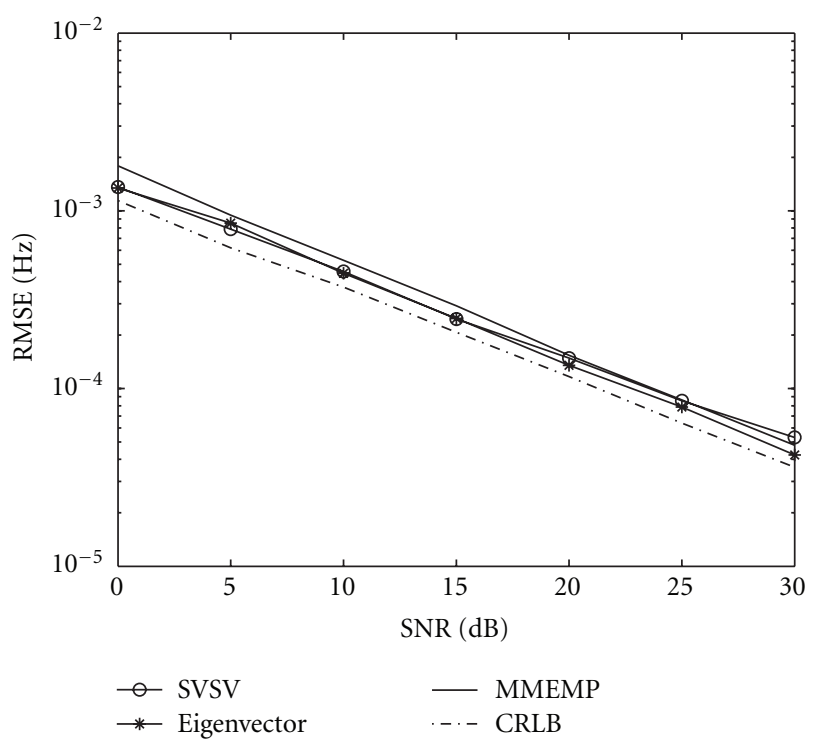

FIGURE 14: RMSE of the estimated frequency $f_{31}$ versus SNR.

-0.00817 to 0.09183 (i.e., the frequency gap $\theta_{2}-\theta_{1}$ varies from -0.05 to 0.05 ), the RMSEs of the frequency estimations are shown in Figure 19. From it, we can see that the proposed algorithm is slightly sensitive to the frequency gap. When the absolute value of the frequency gap is larger than 0.03 , the proposed algorithm performs well.

\section{Conclusion}

A novel two-stage separated virtual steering vector-based algorithm is proposed for $2 \mathrm{D}$ frequencies estimation prob-

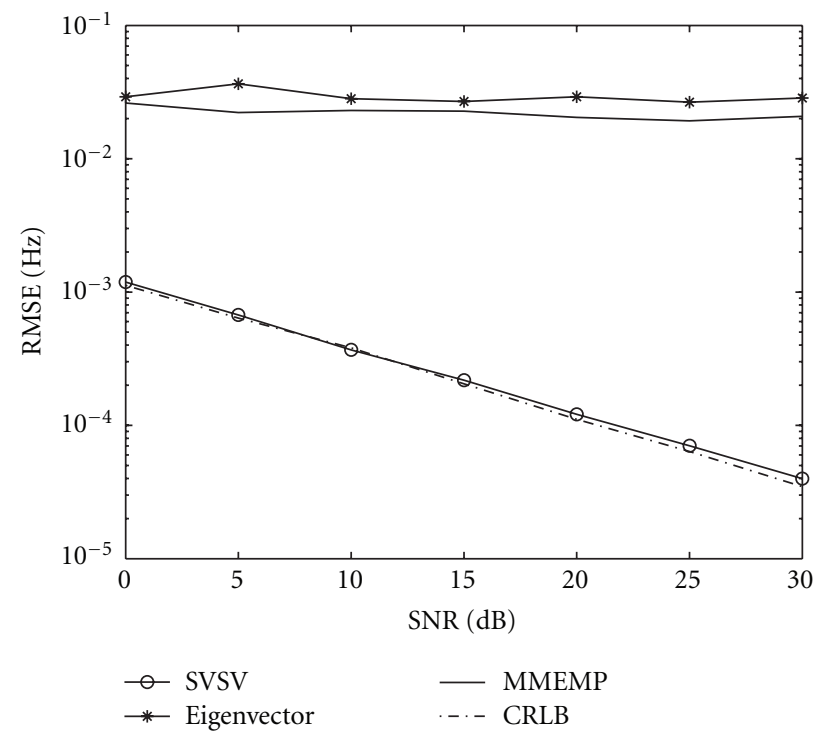

FIGURE 15: RMSE of the estimated frequency $f_{32}$ versus SNR.

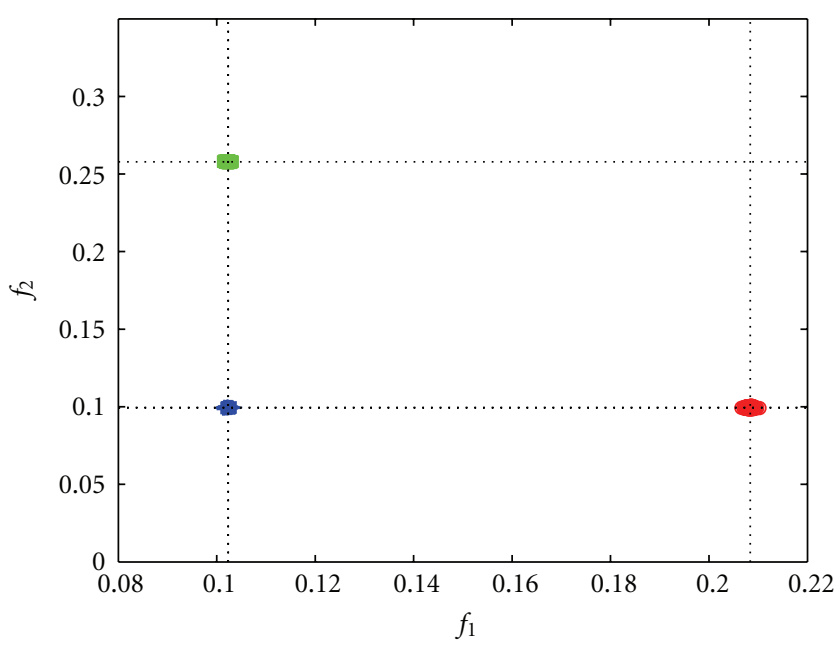

Figure 16: 2D plot of 500 independent estimates for three 2D frequencies using the proposed algorithm.

lem. The algorithm arranges the measurement data as virtual rectangular-shaped data. Moreover, by introducing one special electric angle which is the combination of $2 \mathrm{D}$ frequencies, we derive a rank-reduction propagator method to estimate the introduced electric angle and the first part of separated virtual steering vector. The second part of the separated virtual steering vector is obtained using least-square operator. Finally, 2D frequencies are obtained from the recovered steering vector. Therefore, the resultant SVSV algorithm does not require spectral search or pairing parameters or singular value decomposition (SVD) of data matrix. 


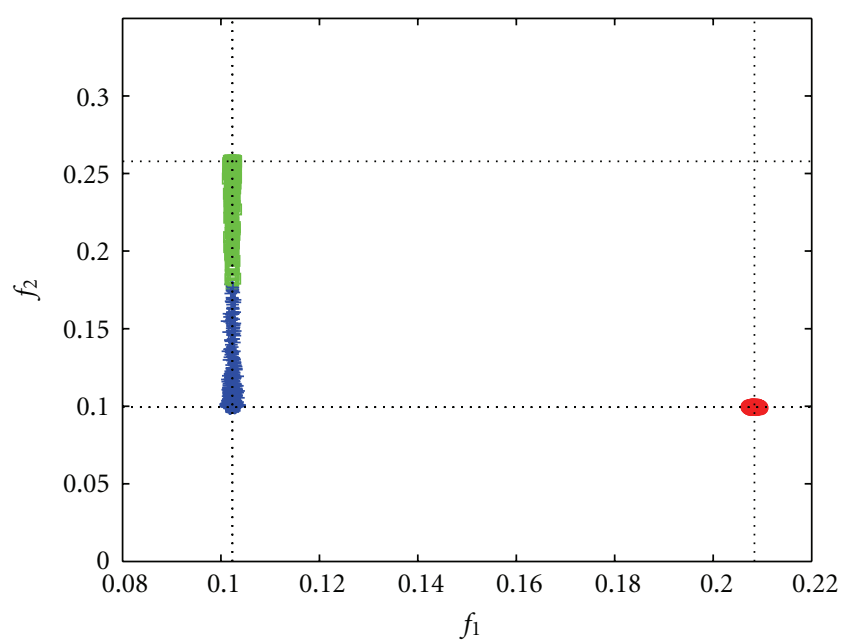

Figure 17: 2D plot of 500 independent estimates for three 2D frequencies using the MMEMP algorithm.

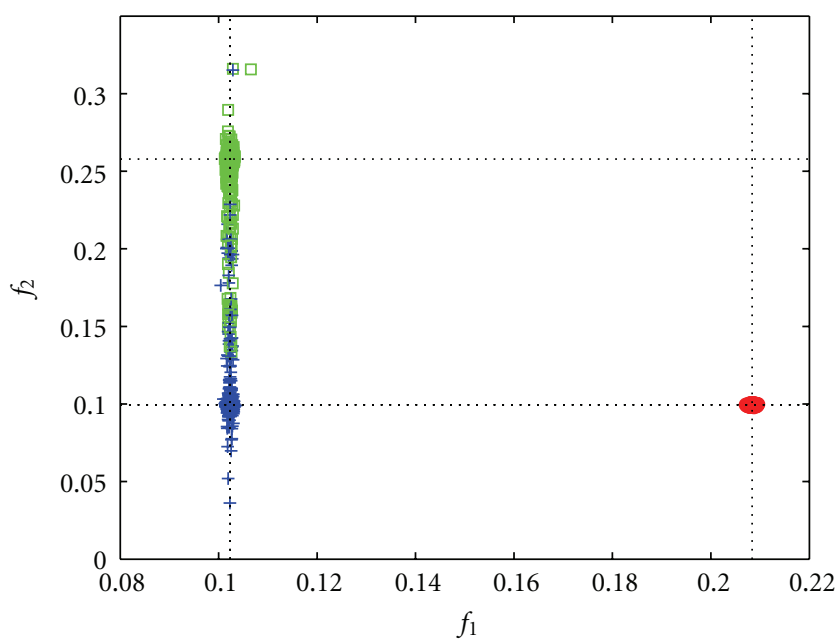

Figure 18: 2D plot of 500 independent estimates for three 2D frequencies using the Eigenvector algorithm.

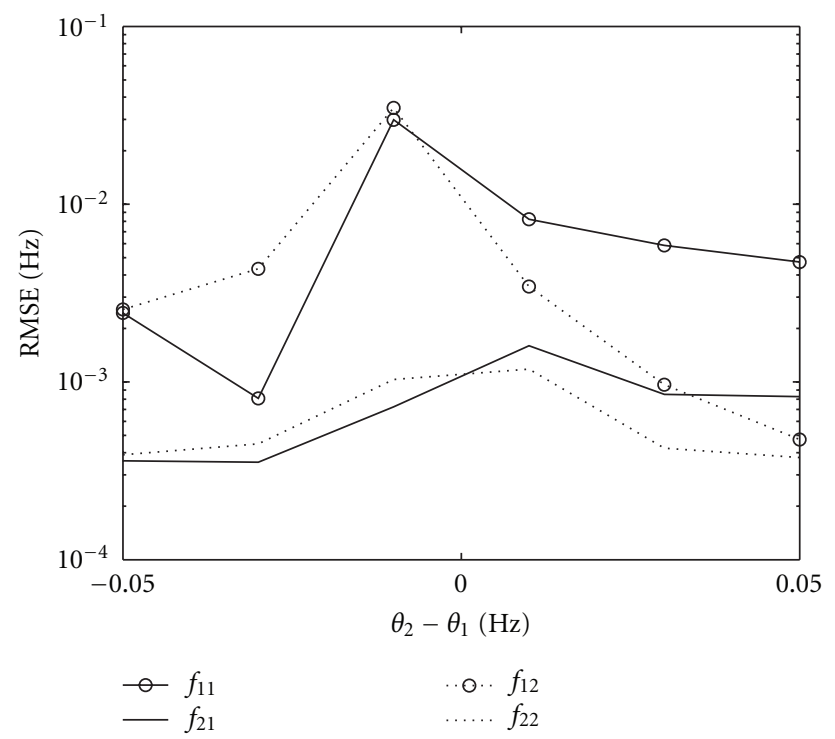

FIGURE 19: RMSE of the estimated frequencies versus varied frequency of $f_{22}$.

\section{Acknowledgments}

This work was supported by the Important National Science and Technology Specific Project under Grant 2009x02011001, by the National Natural Science Foundations of China under grant 60901059 and 61075044, by the China Postdoctoral Science Foundation funded projects under grant 201003679 and 20100481355, by the Educational Department Foundation grant 09JK629 and Natural Science Foundation under grant 2010JQ8001 of Shaanxi Province, and by the Discipline Union Fund under grant 116210905 of Xi'an University of Technology.

\section{References}

[1] J. H. McClellan, "Multidimensional spectral estimation," Proceedings of the IEEE, vol. 70, no. 9, pp. 1029-1039, 1982.

[2] S. L. Marple, Digital Spectral Analysis with Applications, Prentice-Hall, Englewood Cliffs, NJ, USA, 1988.

[3] M. P. Clark and L. L. Scharf, "Two-dimensional modal analysis based on maximum likelihood," IEEE Transactions on Signal Processing, vol. 42, no. 6, pp. 1443-1452, 1994.

[4] M. P. Clark and L. L. Scharf, "A maximum likelihood estimation technique for spatial-temporal modal analysis," in Proceedings of the 25th Asilomar Conference on Signals, Systems \& Computers, vol. 1, pp. 257-261, Pacific Grove, Calif, USA, November 1991.

[5] H. C. So and F. K. W. Chan, "Approximate maximumlikelihood algorithms for two-dimensional frequency estimation of a complex sinusoid," IEEE Transactions on Signal Processing, vol. 54, no. 8, pp. 3231-3237, 2006.

[6] B. F. McGuffin and B. Liu, "An efficient algorithm for two-dimensional autoregressive spectrum estimation," IEEE Transactions on Acoustics, Speech, and Signal Processing, vol. 37, no. 1, pp. 106-117, 1989.

[7] N. Srinivasa, K. R. Ramakrishnan, and K. Rajgopal, "On twodimensional maximum entropy spectral estimation," IEEE Transactions on Signal Processing, vol. 40, no. 1, pp. 241-244, 1992.

[8] S. Kay and R. Nekovei, "An efficient two-dimensional frequency estimator," IEEE Transactions on Acoustics, Speech, and Signal Processing, vol. 38, no. 10, pp. 1807-1809, 1990.

[9] F. Vanpoucke, M. Moonen, and Y. Berthoumieu, "An efficient subspace algorithm for 2-D harmonic retrieval," in Proceedings of the International Conference on Acoustics, Speech, and Signal Processing (ICASSP '94), pp. 461-464, Adelaide, Australia, April 1994.

[10] S. Rouquette and M. Najim, "Estimation of frequencies and damping factors by two-dimensional ESPRIT type methods," IEEE Transactions on Signal Processing, vol. 49, no. 1, pp. 237245, 2001.

[11] Y. Hua, "Estimating two-dimensional frequencies by matrix enhancement and matrix pencil," IEEE Transactions on Signal Processing, vol. 40, no. 9, pp. 2267-2280, 1992.

[12] F. J. Chen, C. C. Fung, C. W. Kok, and S. Kwong, "Estimation of two-dimensional frequencies using modified matrix pencil method," IEEE Transactions on Signal Processing, vol. 55, no. 2, pp. 718-724, 2007.

[13] D. Liu and J. Liang, "A Bayesian approach to diameter estimation in the diameter control system of silicon single crystal growth," IEEE Transactions on Instrumentation and Measurement, vol. 60, no. 4, pp. 1307-1315, 2011. 
[14] J. Liu and X. Liu, "An eigenvector-based approach for multidimensional frequency estimation with improved identifiability," IEEE Transactions on Signal Processing, vol. 54, no. 12, pp. 4543-4556, 2006.

[15] M. Haardt, F. Roemer, and G. Del Galdo, "Higher-order SVD-based subspace estimation to improve the parameter estimation accuracy in multidimensional harmonic retrieval problems," IEEE Transactions on Signal Processing, vol. 56, no. 7, pp. 3198-3213, 2008.

[16] R. Roy and T. Kailath, "ESPRIT-estimation of signal parameters via rotational invariance techniques," IEEE Transactions on Acoustics, Speech, and Signal Processing, vol. 37, no. 7, pp. 984-995, 1989.

[17] R. O. Schmidt, "Multiple emitter location and signal parameter estimation," IEEE Transactions on Antennas and Propagation, vol. 34, no. 3, pp. 276-280, 1986.

[18] S. Marcos, A. Marsal, and M. Benidir, "The propagator method for source bearing estimation," Signal Processing, vol. 42, no. 2, pp. 121-138, 1995.

[19] J. Liang, X. Zeng, W. Wang, and H. Chen, "L-shaped arraybased elevation and azimuth direction finding in the presence of mutual coupling," Signal Processing, vol. 91, no. 5, pp. 1319$1328,2011$.

[20] M. D. Zoltowski, M. Haardt, and C. P. Mathews, "Closed-form 2-D angle estimation with rectangular arrays in element space or beamspace via unitary ESPRIT," IEEE Transactions on Signal Processing, vol. 44, no. 2, pp. 316-328, 1996.

[21] J. Liang and D. Liu, "Joint elevation and azimuth direction finding using 1-shaped array," IEEE Transactions on Antennas and Propagation, vol. 58, no. 6, pp. 2136-2141, 2010.

[22] A. J. Barabell, "Improving the resolution performance of eigenstructure-based direction-finding algorithms," in Proceedings of the IEEE International Conference on Acoustics, Speech and Signal Processing (ICASSP '83), pp. 336-339, Boston, Mass, USA, May 1983.

[23] S. M. Kay, Fundamentals of Statistical Signal Processing: Estimation Theory, Prentice Hall, Upper Saddle River, NJ, USA, 1993. 